\title{
ROLE OF LAPAROSCOPY IN BLUNTABDOMINAL TRAUMA
}

Mohamed A. Yehia M.D., Sami A. Khalifa M.D., Mohamed E. Atia M.D., Wael M., AbdAlla M.D. and

Ramadan M. Ali M.Sc.

General Surgery department, Faculty of Medicine, Zagazig University

\begin{abstract}
Background: Treatment of blunt abdominal trauma requires a precise diagnosis that is not always possible with imaging techniques. The introduction of laparoscopic technique is increasingly used alternative to open surgery. Diagnostic laparoscopy with therapeutic option in stable patients is an emerging technique. Objective: To assess the efficacy of laparoscopy and its role in patients with blunt abdominal trauma. Patients and methods: 40 hemodynamically stable patients with suspicious abdominal injuries underwent diagnostic or therapeutic laparoscopy at the trauma service of Zagazig University Hospital. Inclusion criteria: hemodynamically stable patients with suspicious abdominal injuries. Exclusion criteria: penetrating abdominal trauma, marked hemodynamic instability, Patients with increased intracranial tension and Patients with general or local contra-indications for laparoscopy. Operative technique: creation of Pneumoperitonium with placement of three ports, one 10-mm for the telescope and two 5-mm trocars. If necessary, accessory trocars were inserted. A thorough exploration of the abdominal cavity was carried out. The hemoperitoneum or bowel contents were aspirated, and the lesion causing the injury was located and manged, If possible. Conversion to laparotomy was decided in cases of nonsatisfactory or incomplete abdominal examination Statistical analysis: SPSS version 15. Results: laparoscopic mangement showed results better than that of open exploration as regard postoperative pain, operative time, hospital stay and most of complications. Conclusion: Laparoscopic mangemant of haemodynamically stable blunt trauma is a promising solution which needs increasing its learning curve for optimum results.
\end{abstract}

Keywords: blunt - trauma - laparoscopic.

\section{INTRODUCTION}

$\mathbf{T}$ The decision, in favor of surgery or nonoperative conservative treatment in blunt abdominal trauma, requires a precise diagnosis that is not always possible with imaging techniques. There is great danger that an, injury to the diaphragm or intestines may be over-looked. Indications for exploratory laparotomy have traditionally been generous, to the extent that up to $41 \%$ of exploratory laparotomies turn out to be nontherapeutic ${ }^{(\mathbf{1})}$. A diagnostic laparoscopy with therapeutic option should only be attempted in stable patients. Three trocars are usually used and the abdomen is explored systematically, beginning with the right upper quadrant and continuing clockwise. Hollow viscus injuries and injuries to the diaphragm and mesentery can be detected and sutured laparoscopically. Injuries to parenchymal organs are not a primary focus of laparoscopy, but with a laparoscopic approach, they usually no longer bleed in stable patients and can be sealed with tissue adhesive and collagen tamponade to prevent re-bleeding ${ }^{(2)}$.

The routine use of laparoscopy can achieve a sensitivity of $90-100 \%$ in abdominal trauma. This can reduce the number of unnecessary laparotomies and the related morbidity. ${ }^{(3)}$

Laparoscopy can be performed safely and effectively in stable patients with abdominal trauma. The most important advantages are reduction of the nontherapeutic laparotomy rate, morbidity, shortening of hospitalization, and costeffectiveness. In the future, new developments of equipment can be expected to increase the use of minimally invasive techniques in abdominal trauma cases ${ }^{(4)}$.

The most common indications for laparoscopic diag $\neg$ nosis and treatment:

1. Free fluid of unknown source.

2. Blunt trauma - suspected intestinal injury

3 . Injury to the mesentery.

4. Unclear abdomen after blunt trauma

5. Pancreatic injury.

6. Penetrating trauma - stable patient

The positioning and preparation of the patient for trauma laparoscopy is essentially the same as for a trauma laparotomy. Conversion to conventional open approach to the thorax and abdomen should be possible without delay or additional preparation as for every trauma laparotomy ${ }^{(5)}$.

\section{Objective}

In view of the above, this study was conducted to assess the efficacy of laparoscopy and its role in patients with blunt abdominal trauma.

\section{PATIENTS AND METHODS}

From April 2010 and March 2012, 40 hemodynamically stable patients with suspicious abdominal injuries underwent diagnostic or therapeutic laparoscopy at the trauma service of Zagazig University Hospital. Laparoscopic evaluation was performed only on patients who met the inclusion criteria.

\section{Inclusion criteria}

All stable patients with blunt abdominal trauma -other than those mentioned in the contraindications- were included namely:

- Stable patients with definite occurrence of intraabdominal injury. 
- Patients with equivocal result by clinical and radiological evaluation.

\section{Exclusion criteria}

The following patients will be excluded from our study:

- Stable patients not in need for any intervention for conservative management.

- Patients with penetrating abdominal trauma.

- Patients with marked hemodynamic instability, those cannot tolerate the delays in laparoscopic entry into the abdomen and the pneumoperitonium that laparoscopy requires.

- Patients with definite occurrence of major abdominal injury that cannot be managed laparoscopically e.g. pancereatico-duedenal injury.

- Patients with increased intracranial tension and poly-traumatized patients (more than two organs affected.

- Patients with general or local contra-indications for laparoscopy as decompensated cardiac patients and patients with previous major abdominal surgery expecting marked intra-abdominal adhesions.

\section{Preparation of patients}

Patients consented to diagnostic or therapeutic laparoscopy and exploratory laparotomy whenever needed.

Initially all patient were subjected to proper history taking including: age, sex, mode and time of trauma, concurrent injury, time of last meal and associated medical illness. Then proper general and local examination was conducted. Then all patients were investigated regarding:

- CBC, to be repeated after 6 or 12 hours for selected cases.

- Kidney functions, liver functions, coagulation profile, serum sodium and potassium level and blood sugar level .

- Serum amylase.

- Chest X-ray.

- X-ray abdomen and pelvis; erect and supine.

- Pelvi-abdominal ultrasound .

- Pelvi-abdominal CT.

\section{Operative technique}

Laparoscopic evaluations were performed in the operating room with the patient under general anesthesia by a team with significant experience in emergency laparotomy and laparoscopy. Pneumoperitoneum with carbon dioxide was established via an open technique at the umbilicus, and a forward-viewing laparoscope $\left(30^{\circ}\right)$ was inserted. Two additional 5 or 10 - and $12-\mathrm{mm}$ trocars were placed laterally to the right and left rectus sheath. Intraabdominal pressure was limited to $15 \mathrm{mmHg}$.A thorough exploration of the abdominal cavity was carried out with the surgeon standing at the patient's left side and the patient in the Trendelenburg position .

Inspection of the pelvic structures, including the sigmoid colon, both groins, both iliac regions, and the bladder was done. Thereafter, the right side of the colon was inspected, and the small bowel was examined from the ileocecal valve to the ligament of Treitz, with care taken to inspect both sides of the mesentery carefully.

The transverse colon, splenic flexure, and descending colon were inspected with the surgeon standing at the patient's right side. The liver, stomach, spleen, and diaphragm were inspected with the patient in the reverse Trendelenburg position.

After the abdominal cavity had been examined carefully, the hemoperitoneum or bowel contents were aspirated, and the lesion causing the injury was located. If necessary, accessory trocars were inserted, depending on the site of the lesion. Conversion to laparotomy was decided in cases of nonsatisfactory or incomplete abdominal examination. In the event of active bleeding, hemostasis was performed with diathermy, clips, endoloop-type ligation and suture-ligation.

Postoperatively, the patients were observed for vital data, $\mathrm{Hb}$ level, and return of bowel functions and wound complications. The patients who underwent splenectomy were given vaccination against meningococcal, pneumococcal, and $\mathrm{H}$. influenza type $\mathrm{B}$ infections. The patients were discharged after return of normal bowel functions, drain removal and any complication was ruled out.

\section{Patient assessment}

Postoperative assessment included pain assessment, the mechanism of injury, operative technique, operative time, length of hospital stay and complications. To assess the postoperative pain, visual analogue scale of pain was used with the results drawn on $10 \mathrm{~cm}$ scale ranging from 0 (no pain) to 10 (worst pain).

\section{Statistical analysis:}

Data were coded, entered and analyzed using SPSS version 15.Qui square and t-test were used.

\section{RESULTS}

Demographic data of patients included in the study as regard to age and sex is demonstrated in table (1).

The mechanisms of injury were shown in table (2). The most common causes of BAT are road traffic accident followed by pedestrian accidents, fall from heights and abdominal blows. ISS of some cases, as shown in table (3), was apparently high. This was due to associated extremities and pelvic injuries In spite of the various diagnostic methods currently used 
(abdominal ultrasonography and computed tomography scan), it is difficult to evaluate the presence and severity of intraabdominal injuries as shown in table (4).

Most of cases were injury of liver followed by spleen. Five cases showed only minimal intraperitoneal blood with no definitive injury, table (5).

As shown in table (6), cases completed laparoscopicaly extended up to two hours as in gastric repair. Time of laparoscopic exploration of abdominal cavity ranged from 25 to 35 minutes before decision of conversion. 13 cases were converted to open repair. Small intestinal, uncontrolled splenic and liver injuries were the causes of conversion.
Post operative pain was less in cases completed laparoscopically which is statistically significant, table (7). Pain was controlled by intramuscular diclofenac sodium and replaced by oral ketofen on resuming oral intake.

As shown in table (8) the incidence of postoperative wound infection had much more decreased in completely laparoscopic cases.There is significant difference between the periods of hospital stay of both groups, table (9). Hospital stay extended up to 11 days in open exploration for management of deep infection. Most of hospital stay in laparoscopic group did not exceed 3 days with few cases stayed in hospital up to 8 day.

Table (1): Demographic data

\begin{tabular}{lcccc}
\hline & \multicolumn{2}{c}{$\mathrm{X} \pm \mathrm{SD}$} & \multicolumn{2}{c}{ Range } \\
\hline Age (years) & \multicolumn{2}{c}{$37.93 \pm 10.7$} & & $18-60$ \\
\hline & Male & $\%$ & Female & $\%$ \\
\hline Gender & 22 & 55 & 18 & 45 \\
\hline
\end{tabular}

Table (2): mechanism of injury

\begin{tabular}{lll}
\hline & No. & $\%$ \\
\hline Motor accident & 14 & 35.0 \\
\hline Pedstrian & 10 & 25.0 \\
\hline Fall from height & 8 & 20.0 \\
\hline Physical assault & 5 & 12.5 \\
\hline Sport accident & 3 & 7.5 \\
\hline
\end{tabular}

Table (3): ISS of cases

\begin{tabular}{lll}
\hline ISS & $X \pm S D$ & range \\
\hline- & $38.0 \pm 6.1$ & $20-51$ \\
\hline
\end{tabular}

Table (4): preoperative imaging findings

\begin{tabular}{lll}
\hline \multicolumn{1}{l}{ No. } & $\%$ \\
\hline FAST & & \\
\hline- ve & 16 & 40 \\
\hline+ ve & 24 & 60 \\
\hline CT & & \\
\hline- ve & 11 & 27.5 \\
\hline+ ve & 29 & 72.5 \\
\hline
\end{tabular}


Table (5): Type of intrabdominal injuries

\begin{tabular}{lcccccc}
\hline & \multicolumn{2}{l}{$\begin{array}{l}\text { Non-converted } \\
(\mathrm{n}=27)\end{array}$} & \multicolumn{2}{l}{ Converted $(\mathrm{n}=13)$} & $\mathrm{X}^{2}$ & $\mathrm{P}$ \\
\hline- ve & 5 & 18.5 & 0 & 0.0 & 1.32 & 0.25 \\
\hline Spleen & 5 & 18.5 & 5 & 38.5 & 0.95 & 0.32 \\
\hline Liver & 11 & 40.7 & 5 & 38.5 & 0.02 & 0.89 \\
\hline Small intestine & 0 & 0.0 & 3 & 23.1 & 6.57 & 0.011 \\
\hline Mesenteric & 4 & 22 & 0 & 0.0 & 1.88 & 0.17 \\
\hline stomach & 2 & & & & & \\
\hline
\end{tabular}

Table (6): Duration of laparoscopic management

\begin{tabular}{lcc}
\hline & $\begin{array}{c}\text { Non converted } \\
(\mathrm{N}=27)\end{array}$ & $\begin{array}{c}\text { Converted (laparoscopic time) } \\
\mathrm{N}=13\end{array}$ \\
\hline$\overline{\mathrm{X}} \pm \mathrm{SD}$ & $55.9 \pm 17.4$ & $31.5 \pm 3.1$ \\
\hline Range & $20-125$ & $25-35$ \\
\hline
\end{tabular}

Table (7): Pain assessment in the post-operative period; visual analogue scale of pain (VAP)

\begin{tabular}{lcccc}
\hline & $\begin{array}{c}\text { Non-converted } \\
\mathrm{N}=27\end{array}$ & $\begin{array}{c}\text { Converted } \\
\mathrm{N}=13\end{array}$ & t- test & p-value \\
\hline VAP & $2.93 \pm 0.8$ & $\begin{array}{c}7.31 \pm 0.75 \\
6-8\end{array}$ & 16.1 & $<0.001$ \\
$\chi \pm \mathrm{SD}$ & $2-4$ & & \\
Range & & $6-8$ & \\
\hline
\end{tabular}

Table (8): Wound infections

\begin{tabular}{|c|c|c|c|c|c|c|}
\hline & \multicolumn{2}{|c|}{ Non-converted $(n=27)$} & \multicolumn{2}{|c|}{ Converted $(n=13)$} & \multirow[t]{2}{*}{$\overline{X^{2}}$} & \multirow[t]{2}{*}{$\mathrm{P}$} \\
\hline & $\mathrm{N}$ & $\%$ & $\mathrm{~N}$ & $\%$ & & \\
\hline No. & 26 & 96.3 & 7 & 53.8 & 8.21 & 0.004 \\
\hline$\overline{Y e s}$ & 1 & 3.7 & 6 & 46.2 & & \\
\hline
\end{tabular}

Table (9): hospital stay

Non-converted Converted t- test p-value

\begin{tabular}{lcccc}
\hline Hospital stay (days) & & & & \\
$\chi \pm$ SD & $3.26 \pm 1.65$ & $5.9 \pm 1.0$ & 5.36 & $<0.001$ \\
Range & $1-8$ & $4-11$ & & \\
\hline
\end{tabular}

\section{DISCUSSION}

The abdomen arguably presents the greatest diagnostic and therapeutic challenge among all the zones of injury because it requires an experienced surgical clinician and frequently advanced imaging or invasive procedures for accurate diagnosis and definitive therapy for traumatic injury ${ }^{(6)}$

The most common mechanism of BAT are road traffic accidents followed by pedestrian accidents, fall from heights and abdominal blows. On contrary, Al-Ayoubi et al, ${ }^{(7)}$ reported that fall from height was the most common mechanism, 126 patients of 256 , followed by traffic accident
Clinical abdominal examination is inaccurate for the assessment of the BAT patients since there are often distracting injuries, altered levels of consciousness, non specific signs and symptoms, and large differences in individual patient reactions to intra-abdominal injury ${ }^{(8)}$.

The mean of ISS, in this study, was 38. This is due to associated pelvic and extremity injury. This is consistent with Avarello et al, ${ }^{(9)}$ with ISS of 39.9. It was due to head and pelvic injuries. ISS was 19.3 with Feliz et al. ${ }^{(10)}$.

Due to the recognized inadequacies of physical examination, trauma surgeons have come to rely on a number of diagnostic adjuncts. Commonly used modalities include diagnostic 
peritoneal lavage (DPL) and computed tomography (CT). Focused abdominal sonography for trauma (FAST) has been included in the diagnostic armamentarium of Hoff et al., $\left({ }^{11)}\right.$.

Despite their many positive qualities, all diagnostic methods have some drawbacks. There has been increasing interest in the use of abdominal US because it is portable, noninvasive, rapid, and easily repeatable.. FAST examination is rapidly becoming an accepted practice in many trauma centers. The FAST procedure surveys for blood in the pericardial sac and intraabdominal fluid collection in Morison's pouch, the splenorenal recess, bilateral subphrenic space, bilateral paracolic gutter, and Douglas cul de sac. It is completed in about $2.5 \mathrm{~min}$ However, it definitely is an operator-dependant test, and it is less accurate for diagnosis of diaphragmatic and hollow viscous injury ${ }^{(\mathbf{1 2})}$.

Laparoscopy was first used for a trauma patient in 1956 by Lamy (13) since then, Gazzaniga et al. ${ }^{(14)}$ and Carnevale et al. ${ }^{(13)}$ have noted that laparoscopy is useful for determining the need for laparotomy. Berci et al ${ }^{(\mathbf{1 5})}$ reported that he had reduced the number of nontherapeutic laparotomies performed for hemoperitoneum by $25 \%$ through the use of laparoscopy in 150 patients with blunt abdominal trauma .

In our study, we used U/S for all patients as an initial assessment modality, which is similar to Hoff et al., ${ }^{(11)}$. In practice management guidelines for the evaluation of blunt abdominal trauma who considered FAST as the initial diagnostic modality to exclude hemoperitoneum.

Also, in support to current study, Kendall et al., (16) found out a reported limitation of ultrasound in the setting of blunt trauma was poor specificity for determining the source of hemoperitoneum, as well as a limited ability to detect solid organ injury (SOI) in the absence of free fluid. Tsui and colleagues ${ }^{(17)}$, in BAT, rapid determination of which patients should require emergency laparotomy is crucial for life saving, especially for those with unstable haemodynamics. On the other hand, avoidance of unnecessary laparotomy, which is an invasive procedure with inherent complications, is also important. The FAST scan provides a useful initial diagnostic tool for this kind of patient.

In our work FAST was positive in 24 patients; $60 \%$ and it was negative in $16 \mathrm{pt} ; 40 \%$.

Also, Schnüriger et al., ${ }^{(18)}$ in their clinical study implied that the FAST examination at the primary assessment failed to detect free fluid or organ lesions in 1 of every 5 patients with confirmed spleen or liver injury and they concluded that the low overall diagnostic sensitivity of FAST may lead to under estimated injury patterns and delayed complications may occur. Hence, in hemodynamically stable patients with abdominal trauma, an early CT scan should be considered.

So that, we used CT scan for all patients with blunt abdominal trauma who were stable and cooperative. It was more accurate in diagnosing solid organ injury but missed hollow visceral injuries and this was similar to study of Banz et al., (19) who stated that CT exhibits very high sensitivity and specificity in detecting the majority of solid organ injuries, but unfortunately misses up to $15 \%$ of small bowel and mesenteric injuries as well as some acute pancreatic injuries, also Lee et al., ${ }^{(20)}$ considered CT was the definitive technique because of its high sensitivity and specificity in injury detection, localization, and grading. However, CT may not be an option for those patients who are clinically unstable to travel to the CT scanner, who are pregnant, or who will not fit in the scanner due to their body habitus. Sonography has some specific advantages over $\mathrm{CT}$ in that it is a bedside examination, is a relatively expedient examination, and uses nonionizing radiation. Furthermore, patients undergoing sonography do not require contrast agents and thus are spared the associated risk of contrast reaction and nephrotoxicity.

In our series Pelvi-abdominal CT was positive in 21 patients; 16 pt had intra-abdominal collection and 5 patients had evident organ injury, and it was negative in 19 patients .

In the evaluation and management of the abdominal injury, current diagnostic methods have a defined sensitivity, specificity, and accuracy, but none of these represents a gold standard. Thus abdominal exploration by laparotomy should not be discarded as a worthy diagnostic and therapeutic procedure for patients with equivocal and unreliable findings. It is associated with complication rates as high as $40 \%$ including a $10 \%$ to $40 \%$ negative laparotomy rate, a $20 \%$ morbidity rate, a $0 \%$ to $5 \%$ mortality rate and a 3\% long-term risk of bowel obstruction ${ }^{(21) .}$

Whereas, inspection of the abdominal parities and solid viscera is relatively easy to perform, complete examination of the intestine presents a greater challenge, with a 5\% missed injury rate per patient. Careful and complete inspection of the bowel and its mesentery is imperative if laparoscopic trauma examination is to be credible (12).

In the current study, 40 patients with blunt abdominal trauma, haemodynamically stable, underwent diagnostic laparoscopy. Five patients were negative whereas thirteen patients were 
converted to conventional open exploration. Most of the converted cases were small bowel and splenic injuries .

We used laparoscopy as diagnostic and therapeutic tool in the managment of blunt abdominal trauma and we found it helpful and were consistent with Prasad and Agarwal (22) who confirmed that the role of laparoscopy in blunt trauma has yet to be defined. In experienced hands, it has been shown to reduce the negative laparotomy rate and identify and treat diaphragmatic and visceral injuries. In our study 5 patients from those subjected to laparoscopy were negative and avoided negative laparotomies, whereas 22 patients benefits from therapeutic intervention and 13 patients from diagnostic. Johnson et al, ${ }^{(23)}$ reported that, over the 10 -year study period, 22 patients sustaining blunt trauma underwent diagnostic laparoscopy. Laparoscopy was negative in $4(18.2 \%)$ patients and nontherapeutic in $15(68.2 \%)$ patients. Three patients $(13.6 \%)$ required conversion to an open procedure

This also was consistent with Choi and Lim, (24). They found out that diagnostic and therapeutic laparoscopy applied to carefully select hemodynamically stable trauma patients proved to be safe and technically feasible. It also reduced the negative and nontherapeutic laparotomies and offered profound therapeutic potential and cost effectiveness. In addition, Sitnikov et al., ${ }^{(25)}$ in their study concluded that, diagnostic and therapeutic video assisted laparoscopy can confidently be used in trauma centers as a diagnostic and therapeutic technique in patients with small bowel injuries (SBIs). This technique has demonstrated significant accuracy, specificity, and sensitivity in triage and management of SBIs patients. Hence it allows early diagnosis of bowel injury, decreasing the time to definitive repair, and when combined with therapeutic laparoscopy reduces morbidity, mortality, hospital costs, and length of hospitalization associated with nontherapeutic open laparotomy and their complications in patients with SBIs.

It is likely that the continued evolution of minimally invasive surgery will provide additional lessons that will challenge traditional concepts and change the way patients are cared for in the future ${ }^{\text {(26). }}$

The average hospital stay for the group that underwent totally therapeutic laparoscopy $(n=27)$ was 3.2 days, but the stay for the nontherapeutic (converted) laparoscopy group $(\mathrm{n}=13)$ was 5.9 days. There were significant difference between the hospital stay with advantage for laparoscopy due to less pain and early recovery. This was consistent with Johnson et al, ${ }^{(23)}$. The mean length of hospital stay, in this study, for patients with blunt injuries was 9 days for the laparoscopyonly group compared with 20 days for those requiring laparotomy

One patient in the totally laparoscopic cases had wound infection that involved the umbilical port site and 6 patients had wound infection in converted cases. The management was conservative by antibiotic and opening the wound. Another patient had paralytic ileus that lasted for 5 days and subsided spontaneously with conservative management by intra-venous fluid and electrolyte balance. And the last patient had atelectasis that had manifested on the third day by fever, cough and decreased air entry on left lower lung segment. The patient was managed conservatively by chest exercise and third generation cephalosporin. All the recorded complications have reported to occur more frequently with open laparotomy than in laparoscopic surgery .

There were two missed injuries, and no mortality occurred in the series, so laparoscopy can be considered safe with regard to the minimal morbidity, high sensitivity and specificity observed in our study.

Conclusion: Laparoscopic mangemant of haemodynamically stable blunt trauma is a promising solution which needs increasing its learning curve for optimum results.

\section{REFERENCES}

1- Shanmuganathan K, Mirvis SE, Chiu WC, Killeen KL, Hogan GJF and Scalea TM (2004): Penetrating torso trauma: triple-contrast helical CT in peritoneal violation and organ injury - a prospective study in 200 patients. Radiology; 231:775-84.

2- Chol YB and Lim KS (2003): Therapeutic laparoscopy for abdominal trauma. Surg Endosc ; 17:421-7.

3- Gorecki PJ, Cottam D, Angus LD and Shaftan GW (2002): Diagnostic and therapeutic laparoscopy for trauma: a technique of safe and systematic exploration. Surg Laparosc Endosc Percutan Tech; 12:195-8.

4- Leppaniemi A and Haapiainen R (2003): Diagnostic laparoscopy in abdominal stab wounds: a prospective, randomized study. J Trauma; 155:63645.

5- Taner AS, Topgul K, Kucukel F, Demir A and Sari S (2004): Diagnostic laparoscopy decreases the rate of unnecessary laparotomies and reduces hospital costs in trauma patients. J Laparoendosc Adv Surg Tech A; 11: 207-12.

6- Bonatti Hugo and Calland J F (2008): Trauma Emerg Med Clin N Am 26, 625-648.

7- Fawzi al-Ayoubi, Helen Eriksson, Pur Myrelid, Conny Wallon and Peter Andersson (2012): Distribution of emergency operations and trauma in 
a Swedish hospital: need for reorganization of acute surgical care? Scandinavian Journal of Trauma, Resuscitation and Emergency Medicine, 20:66

8- Alexandrescu R, Sarah J O'Brien, and Fiona E Lecky (2009): A review of injury epidemiology in the UK and Europe: some methodological considerations in constructing rates BioMed Central Public Health, 9:226.

9- Avarello JT, and Cantor R M., (2007): Pediatric Major Trauma: An Approach to Evaluation and Management. Emerg Med Clin N Am 25: 803-83.

10- Alexander Feliz, Barbara Shultz, Chris McKenna and Barbara A. (2006): Diagnostic and therapeutic laparoscopy in pediatric abdominal trauma. J Pediatr Surg. Jan; 41(1):72-7.

11- Hoff W S., Holevar M, Kimberly K. Nagy, Lisa Patterson, Jeffrey S. Young, Abenamar Arrillaga, Michael P. Najarian, and Carl P. Valenziano (2001): practice management guidelines for the evaluation of blunt abdominal trauma Eastern Association for the Surgery of Trauma.

12- Ivatury RR, Simon RJ and Stahl WM (1993): A critical evaluation of laparoscopy in penetrating abdominal trauma. J Trauma; 1993, 34: 822-829.

13- Carnevale N, Baron N and Delany HM (1997): Peritoneoscopy, as an aid in the diagnosis of abdominal trauma: a preliminary report. J Trauma 1997; 17: 634-641

14- Gazzaniga AB, Slanton $\mathrm{WW}$ and Bartlett $\mathrm{RH}$ (1993): Laparoscopy in the diagnosis of blunt and penetrating injuries to abdomen. Am J Surg 1996; 131: 315-31833. 39

15- Berci G, Sackier JM and Paz-Parlow M (1991): Emergency laparoscopy. Am J Surg 1991; 161: 332-335

16- Kendall John L., Jeffrey Faragher, Gwendolyn J. Hewitt, Gregory Burcham and Jason S. Haukoos (2009): Emergency Department Ultrasound Is not a Sensitive Detector of Solid Organ Injury Western Journal of Emergency Medicine Volume X, No. 1: February.

17- Tsui Chi Leung, Hin Tat Fung, Kin Lai Chung and Chak Wah Kam (2008): Focused abdominal sonography for trauma in the emergency department for blunt abdominal trauma Int J Emerg Med 1:183-187
18- Schnüriger Beat, Joachim Kilz, Daniel Inderbitzin, Miranda Schafer, Ralph Kickuth, Martin Luginbühl, Daniel Candinas, Aristomenis K Exadaktylos and Heinz Zimmermann (2009): The accuracy of FAST in relation to grade of solid organ injuries: A retrospective analysis of 226 trauma patients with liver or splenic lesion BMC Medical Imaging, 9:3 doi:10.1186/1471-2342-9-3.

19- Banz Vanessa M, Muhammad U Butt, Heinz Zimmermann, Victor Jeger and Aristomenis K Exadaktylos (2009): Free abdominal fluid without obvious solid organ injury upon CT imaging: an actual problem or simply over-diagnosing? Scandinavian Journal of Trauma, Resuscitation and Emergency Medicine, 3:10.

20- Lee Brett C., Eleanor L. Ormsby, John P. McGahan, Giselle M. Melendres, John R. Richards (2007): The Utility of Sonography for the Triage of Blunt Abdominal Trauma Patients to Exploratory Laparotomy AJR:188, February

21- Roos JE, Willmann JK, Platz A, Pfammatter T, Hilfiker PR and Marincek B. (2002) Multidetector $\mathrm{CT}$ : detection of active hemorrhage in patients with blunt abdominal trauma. AJR Am J Roentgenol; 179(2), 437-444.

22- Prasad A and Agarwal N (2009): Laparoscopic splenectomy in a case of blunt abdominal trauma $\mathbf{J}$ Minim Access Surg. Jul-Sep; 5(3): 78-81.

23- Johnson J., Tabitha G., Alexander R., Joseph B. et al., (2013): The use of laparoscopy in the diagnosis and treatment of blunt and penetrating abdominal injuries: 10-year experience at a level 1 trauma center. Am J Surg.; 205 (3):317-20.

24- Choi Y. B. and Lim K. S. (2002): Therapeutic laparoscopy for abdominal trauma Presented at the 8th World Congress of Endoscopic Surgery, New York, New York, USA, 13-16 March

25- Sitnikov V, Abdulkadir Yakubu, Vagan Sarkisyan and Michail Turbin (2009): The role of videoassisted laparoscopy in management of patients with small bowel injuries in abdominal trauma Surg Endosc 23:125-129.

26- Ellison E. Christopher and Carey L C. (2008): Lessons Learned from the Evolution of the Laparoscopic Revolution Surg Clin N Am 88, 927941. 